\title{
TECNOLOGÍAS EMERGENTES EN LA EDUCACIÓN: UNA EXPERIENCIA DE FORMACIÓN DE DOCENTES QUE FOMENTA EL DISEÑO DE AMBIENTES DE APRENDIZAJE
}

\author{
OsCAR RAFAEL Boude Figueredo*
}

\begin{abstract}
RESUMEN: Este texto describe los resultados obtenidos en una experiencia de formación de docentes, que son estudiantes de la Maestría en Informática Educativa de la Universidad de La Sabana, dentro de la asignatura electiva Tecnologías Emergentes en la Educación, que cuyo objetivo es: "ofrecer al estudiante un espacio de reflexión y conceptualización sobre las principales tecnologías emergentes (TE) y sus usos en la educación". Entre los principales resultados, se encuentra que los estudiantes analizaron, indagaron y reflexionaron sobre las siguientes TE: Computación en la Nube, Inteligencia Colectiva, Mashups de Datos, Webs en Colaboración y Redes sociales. Asimismo, propusieron definiciones desde el punto de vista académico de cada una de ellas y diseñaron ocho ambientes de aprendizaje que integran de forma pedagógica una o varias TE en diferentes contextos educativos.
\end{abstract}

Palabras clave: Tecnologías emergentes. Trabajo colaborativo. Estrategias didácticas. Tecnologias de la información y la comunicación. Construcción colectiva de conocimiento.

\section{EMERGING TECHNOLOGIES IN EDUCATION:}

A TEACHER TRAINING EXPERIENCE THAT ENCOURAGES THE DESIGN

OF LEARNING ENVIRONMENTS

ABSTRACT: This paper describes the results of a teacher training experience with a group of students taking the elective Emerging Technologies in Education (in the Maestría en Informática Educativa course at Universidad de La Sabana), whose aim is to "offer students a space for reflection and conceptualization on main emerging technologies (ET) and their uses in education". Among the main results, we find that students analyze, investigate and reflect on the following ETs: Cloud Computing, Collective Intelligence, Data Mashups, Collaborative Webs and Social Networks. They also proposed definitions for each of these ETs from the academic point of view,

Maestría en Informática Educativa y coordinador académico de la Universidad de La Sabana, Colombia. E-mail: oscarbf@unisabana.edu.co 
and designed eight learning environments that integrate, in a pedagogical way, one or more ETs in different educational contexts.

Key words: Emerging technologies. Collaborative work. Teaching strategies. Information and communications technology. Collective construction of knowledge.

\section{TECHNOLOGIES ÉMERGENTES DANS L'ENSEIGNEMENT:}

UNE EXPÉRIENCE DE FORMATION D'ENSEIGNANTS QUI CONSTRUIT

LE PANORAMA D'ENVIRONNEMENTS D'APPRENTISSAGE

RÉSUMÉ: Ce texte décrit les résultats obtenus dans une expérience de formation d'enseignants, ceux qui sont des étudiants du Master en Informatique Éducative de l'Université de La Sabana, à l'intérieur du sujet les Technologies Émergentes dans l'Éducation, dont l'objectif est: “offrir à l'étudiant un espace de réflexion et une conceptualisation sur les principales technologies émergentes (TE) et leurs utilisations dans l'enseignement". Entre les principaux résultats, ils se trouvent que les étudiants ont analysé, recherché et réfléchi sur les aspects suivants: Informatique en Nuages, Intelligence Collective, Mashups de Données, Webs en Coopération et Réseaux Sociaux. De cette forme, ils ont proposé des définitions selon le point de vue académique à chacune d'entre celles-ci et ont développé huit environnements d'apprentissage qui intègrent de forme pédagogique une ou plusieurs TE dans de différents contextes éducatifs.

Mots-clés: Technologies émergentes. Travail colaboratif. Stratégies didactiques. Technologies des informations et de la communication. Construction collective de connaissance.

\section{Introdución}

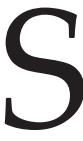

e podría afirmar que el mundo vive hoy un rápido proceso de transformación gracias a los adelantos que durante los últimos años se han alcanzado en los medios de comunicación, la electrónica, las autopistas de la información y los computadores (BOUDE FIGUEREDO, 2008). Una transformación que afecta la forma como nos organizamos, trabajamos, relacionamos y aprendemos (MARCELO, 2001). Una sociedad que se caracteriza por su estructura en red (CASTELLS, 1997), por la abundante y constante circulación de información (HARGREAVES, 2003), que exige ciudadanos con habilidades y competencias que les permitan "manipular y actualizar el conocimiento, seleccionar lo apropiado a cada contexto, aprender y comprender de manera permanente, de tal forma que pueda adaptarlo a situaciones nuevas y de rápido cambio" (PROYECTO TUNNING, 2003). Competencias y habilidades que las instituciones de educación deben contribuir a desarrollar en los ciudadanos durante los diferentes años que dure su proceso de formación.

Lo anteriormente expuesto trae consigo una serie de retos para las instituciones de educación - básica, media y superior -, así como a sus profesores, quienes 
siendo en el mejor de los casos inmigrantes digitales, deben diseñar estrategias didácticas y ambientes de aprendizaje mediados por Tecnologías de la Información y la comunicación (TIC) para contribuir a que sus estudiantes - nativos digitales - desarrollen las competencias requeridas por la sociedad del siglo XXI. Lo cual implica no solo que el docente debe poseer las habilidades tecnológicas necesarias para usar e integrar diversas herramientas tecnológicas a los procesos de enseñanza aprendizaje, sino también que éste debe transformar sus concepciones pedagógicas acerca del ¿Qué?, ¿Cómo? Y ¿Para qué? enseña.

Es decir, comprender tal y como indica Coll (2008) que la capacidad de transformación y mejora que tienen las TIC en la educación radica en el potencial que tienen estas y que puede o no hacerse realidad en función del contexto en donde sean utilizadas, así como de las intenciones que con estas tenga el docente. Con lo cual, la planeación y diseño de un ambiente de aprendizaje mediado por TIC resulta ser un proceso en donde el docente pone en juego sus competencias, habilidades y conocimientos sobre el contexto de sus estudiantes, para determinar según sus intenciones pedagógicas cuál o cuáles son las funciones que las tecnologías deben cumplir dentro de su ambiente de aprendizaje.

No obstante, este debe ser consciente de que cada vez que él y sus estudiantes participan en un ambiente de aprendizaje mediado por TIC, lo hacen a partir de sus imaginarios sobre lo que significa enseñar y aprender, de las TIC, así como del papel que deben jugar en las tecnologías en este espacio; en este sentido los dos ponen en juego sus saberes, concepciones y prácticas que en muchas ocasiones son contradictorios y generan tensiones en el ambiente (JARAMILLO y otros, 2005), tensiones que el docente debe saber reconocer y comprender evitando así posibles frustraciones que le impidan a él y a sus estudiantes alcanzar los objetivos propuestos dentro del ambiente.

Sin embargo, estos son solo algunos de los retos con los que deben enfrentarse los docentes hoy, pues la velocidad con la que se presentan los diferentes adelantos tecnológicos exige profesores dispuestos a estar permanente en procesos de formación y capacitación, a transformar y reflexionar constantemente sobre sus prácticas docentes y a desarrollar competencias en TIC (UNESCO, 2008).

Asimismo, docentes capaces de hacer un alto en el camino, tomar conciencia de los retos e implicaciones que tiene formar a los futuros ciudadanos de la sociedad del conocimiento, reconocer que integrar las TIC a sus procesos de enseñanza y aprendizaje surge como producto de un proceso de reflexión en donde se tiene en cuenta el contexto educativo, las competencias a desarrollar y el diseño curricular, pero, sobre todo, docentes dispuestos a estimular a sus estudiantes para que estos sean el centro del proceso. 
Consciente de estas necesidades, el Centro de Tecnologías para la Academia de la Universidad de La Sabana diseño dentro de su programa de Maestría en Informática Educativa un curso electivo denominado Tecnologías Emergentes en la Educación, cuyo fin es ofrecer a los profesores que toman la asignatura un espacio de reflexión y conceptualización sobre las principales Tecnologías Emergentes (TE) y su proceso de integración en la educación (MAESTRÍA EN INFORMÁTICA EDUCATIVA, 2010), en donde, de forma colaborativa, se diseñen diferentes estrategias didácticas que integren una o varias TE en sus procesos de enseñanza y aprendizaje.

\section{Contexto educativo}

La Maestría en Informática Educativa, a diferencia de la mayoría de programas de postgrados de la Universidad de la Sabana, es un programa de educación superior que no se encuentra suscrito a una facultad. Sin embargo, esta suscrito al Centro de Tecnologías para la Academia de la misma Universidad, la cual es un área académica transversal que tiene funciones de docencia, investigación y proyección social. Ofrece programas de formación en pregrado, postgrado y educación continuada orientados a contribuir tanto en los docentes como en los estudiantes al desarrollo de competencias en TIC, asimismo, está encargada de diseñar las políticas institucionales en cuanto a la integración de TIC a los procesos académicos de la Universidad (CENTRO DE TECNOLOGÍAS PARA LA ACADEMIA, 2010).

El programa tiene como objeto de estudio la Informática Educativa, entendida esta como: "una interdisciplina que estudia no solo las relaciones entre la Informática y la Educación, sino las transformaciones que se generan como producto de la sinergia entre ellas, tanto a nivel práctico como teórico" (CHIAPPE; BOUDE FIGUEREDO, 2010).

Pretende responder a las necesidades del país en cuanto a la formación de docentes e investigadores de educación básica, media y superior en informática educativa, que diseñen proyectos de integración de TIC en sus contextos educativos y que contribuyan a los objetivos planteados por el Ministerio de Educación de Colombia en cuanto a integración de TIC en contextos educativos en plan decenal de educación (MEN, 2006).

Para esto, ha planteado un diseño curricular orientado a fomentar "la reflexión, entendida como un proceso metacognitivo encaminado al mejoramiento continuo como profesional (docente, diseñador o administrador) y como ser humano" (JARAMILLO; CHIAPPE; SANCHÉZ, 2007, p. 27) y a que los estudiantes relacionen sus conocimientos, creencias y experiencias previas con los aprendizajes 
adquiridos en el programa y, a partir de esta relación, diseñen e implementen acciones concretas que pueden involucrar en su práctica docente, encaminadas abeneficiar su contexto educativo.

No obstante, comprende que el proceso de formación docente en integración de tecnologías implica, como bien lo indican Llorente (2008), Hinojo et al. (2002), González y González (2007) y Salinas (2004), el reconocimiento de que cada profesor tiene una concepción de lo que implica aprender y enseñar a través de las TIC, y que dicha concepción junto con las experiencias vividas por estos en el proceso de integración de las TIC a su práctica docente facilita o dificulta el desarrollo de las competencias planteadas en el programa.

Por lo que, cada puesta en escena de los profesores del programa se vuelve un escenario en donde el estudiante aprende tanto del diseño curricular planteado, como de las estrategias de integración de lasTIC utilizadas en cada asignatura.

Asimismo, dentro del programa han sido diseñados diferentes cursos electivos para cada una de las líneas de énfasis del programa, que pretenden complementar el proceso de formación de los estudiantes, y brindarles escenarios de profundización sobre las tendencias actuales de cada línea. Particularmente, para la línea de Integración de las TIC en los ambientes de aprendizaje se diseñaron las electivas de E-learning, Tecnologías emergentes en la educación y Ambientes presenciales y virtuales de aprendizaje, entre otras.

El curso Tecnologías emergentes en la educación ofrece al estudiante una visión general sobre las principales tecnologías emergentes y su integración en la educación. En cada período académico que la asignatura es orientada se abordan cinco TE diferentes. Para esto se toma como referencia las tecnologías identificadas por el New Media Consortium en el informe Horizon desde el 2007 hasta hoy. Ya que dicho informe no solo es un referente mundial al respecto, sino también porque cada día son más los docentes que se acercan a éste buscando herramientas que contribuyan a enriquecer sus ambientes de aprendizaje.

En cada período académico según sus preferencias los estudiantes que toman la asignatura seleccionan cinco de las posibles tecnologías emergentes planteadas en el informe Horizon en los años anteriores, esto debido a que la asignatura está diseñada para que el estudiante sea el centro del proceso, por lo tanto, debe responder a las necesidades de los estudiantes y no a las del docente.

Particularmente en el período académico 2010-2012 el curso fue tomado por diez estudiantes que, durante las 32 horas de clase presencial distribuidas en ocho sesiones de cuatro horas, fueron orientados por el docente para realizar la aproximación teórica y propositiva sobre las siguientes tecnologías emergentes: Computación 
en la Nube, Inteligencia Colectiva, Mashups de Datos, Webs en Colaboración y Redes Sociales. Cabe anotar que el tiempo total de trabajo realizado por los estudiantes, tomando en cuenta el destinado a las actividades de trabajo independiente, fue de 128 horas. Las competencias planteadas para esta asignatura son:

- Reconocer las principales tecnologías emergentes que están siendo utilizadas en la educación.

- Diseñar una estrategia didáctica que involucre una o varias de las tecnologías emergentes abordadas en la asignatura.

- Participar activamente en la solución de problemas en torno a la integración pedagógica de las tecnologías emergentes propuestas.

- Reflexionar alrededor de los diferentes aspectos pedagógicos involucrados al integrar las tecnologías emergentes en la educación

Para lograr que los estudiantes - profesores que toman el programa de postgrado - desarrollen las competencias planteadas en la asignatura, el diseño curricular de la asignatura se dividió en dos partes: en la primera de ellas, se plateó un ambiente de aprendizaje mediado por TIC centrado en el estudiantes, que fomenta la gestión y transformación del conocimiento a partir de la reflexión personal y la construcción colectiva de conocimiento por parte de los estudiantes. La segunda parte de la asignatura se realizó luego del proceso de construcción colectiva de conocimiento. Esta parte se diseño para que, a través de un proceso de reflexión y reconocimiento de sus contextos educativos, cada profesor diseñara una estrategia didáctica que involucrará una o varias de las tecnologías abordadas en la asignatura.

\section{Ambientes de aprendizaje propuestos}

Tal y como se expuso en el apartado anterior, el diseño curricular de la asignatura se dividió en dos partes: para la primera se diseñó un ambiente de aprendizaje mediado por TIC cuyo objetivo fue Contribuir al desarrollo de las competencias planteadas en la asignatura, a través de la reflexión personal y la construcción colectiva de conocimiento alrededor de las Tecnologías Emergentes.

Para esto se definieron como actores dentro del ambiente de aprendizaje a los estudiantes y el profesor, y como elementos del mismo a las TIC e internet. Cabe aclarar en este momento que la distinción entre las TIC e internet como elementos independientes se debe a las funciones que cumplieron las mismas dentro del ambiente de aprendizaje tal y como se puede observar en la siguiente figura: 


\section{Figura 1}

Ambiente de aprendizaje diseñado para la primera parte de la asignatura

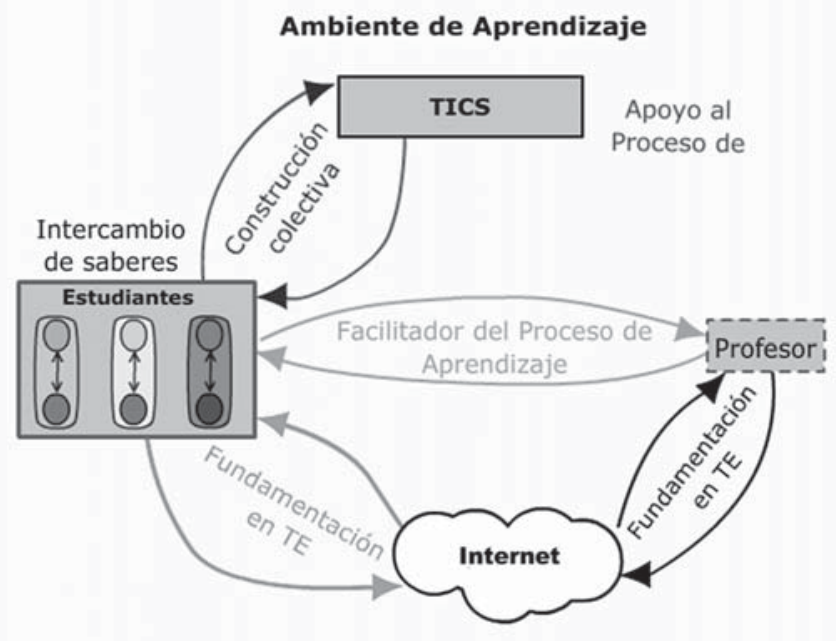

En él, intervienen los estudiantes - en algunos momentos de forma individual y en otros distribuidos en parejas - y el profesor, como actores - agentes dinamizadores - del proceso, que aportan sus conocimientos, concepciones, miedos y percepciones sobre lo que significa enseñar y aprender con las TIC; así como las TIC e Internet como elementos - agentes pasivos - que proveen las herramientas necesarias para la interacción, la comunicación, el intercambio, la conceptualización y la construcción colectiva de conocimiento por parte de los estudiantes.

Ahora bien, con el fin de lograr el objetivo del ambiente de aprendizaje, se diseño una estrategia didáctica basada en la construcción colectiva de conocimiento y en la teoría de espiral de transformación de conocimiento propuesta por Nonaka y Takeuchi (1999), ya que ésta teoría plantea que el conocimiento en los seres humanos se transforma siguiendo un ciclo, que parte del conocimiento tácito que hay en cada persona - conocimientos previos - y que, en la medida en que estos socialicen su conocimiento tácitos haciéndolos explícitos, el conocimiento tácito individual inicial se volverá conocimiento explicito colectivo, el cual será interiorizado por cada persona y en ese proceso se transformaran los conocimientos tácitos iniciales, mezclándolos con los conocimientos nuevos adquiridos.

\section{Estrategia didáctica para la reflexión y construcción colectiva de conoci- miento alrededor de las TE}

Como su nombre lo índica, esta estrategia tiene como fin que los estudiantes reflexionen y construyan de forma colectiva un marco de conocimientos sobre cada 
una de las TE que se abordan en el curso. Para esto se han planteado cuatro fases diferentes denominadas: Conocimientos Previos, Conceptualización Inicial, Construcción Colectiva y Conclusión y Prospectiva.

La fase de Conocimientos Previos está diseñada para que, a partir de los conocimientos previos de los estudiantes, se construya un mapa mental en donde queden plasmadas las concepciones que tienen estos alrededor de cada una de las TE abordadas en el curso. Para esto, se plantearon dos actividades. La primera de ellas es un foro de discusión en donde se les pide a los estudiantes dar respuesta a dos preguntas iniciales. La segunda actividad toma como punto de partida las respuestas dadas por los estudiantes para identificar los conceptos claves, a partir de este momento se fomenta una discusión en el grupo que tiene como fin elaborar un mapa mental en el que queden plasmadas las concepciones del grupo alrededor de cada TE.

La fase de Conceptualización Inicial está diseñada, como su nombre lo indica, para realizar una conceptualización inicial sobre cada una de las TE. Inicialmente se planteó que esta fase se llevará a cabo por parte del profesor-autor de este trabajo como una clase magistral. Sin embargo, luego de la primera sesión de clases los estudiantes solicitaron al profesor cambiar la actividad, pues ellos venían acostumbrados en la maestría a construir sus conocimientos, lo cual le pareció adecuado al profesor-autor. Por esta razón, las actividades planteadas en esta fase se modificaron, para permitir que los estudiantes construyeran sus primero conceptos alrededor de cada TE con la orientación del docente.

La fase de Construcción Colectiva, esta es la fase más larga dentro de todo el proceso, tiene una duración de 15 días para cada TE. Pretende que los estudiantes tomen como punto de partida el trabajo realizado en la fase anterior, para elaborar un blog sobre cada TE. En esta fase el profesor no tiene ninguna interacción con los estudiantes, solo con los líderes de cada temática para proveerles los recursos que necesiten, puesto que estos tienen la responsabilidad de diseñar e implementar una estrategia que les permita construir un Blog de forma colaborativa, en donde se aborde la TE que seleccionaron desde su conceptualización como tecnología, sus alcances como una tecnología que pueda utilizarse en la educación, las ventajas y desventajas que tiene en la educación, y los posibles usos que se pueden dar a la misma.

La última fase se denomina Conclusión y Prospectiva. En esta fase los líderes de cada TE asumen el rol de coordinadores de la misma, presentan al grupo los aportes realizados por todos dentro del Blog. Se discute y concluye sobre los alcances de cada TE en la educación y se plantean algunos ejemplos de cómo cada TE podría integrarse a los procesos de enseñanza y aprendizaje.

Por su parte, el profesor asume en esta fase un doble rol: el primero es comportarse como un estudiante y contribuir a la discusión de la temática; el segundo 
es de orientador de la temática, puesto que en algunos momentos la discusión se desvía del cauce original y es función de él volver a encausarla. En la figura 2 se presenta la estrategia diseñada.

\section{Figura 2}

Estrategia diseñada para la reflexión y construcción colectiva de conocimiento alrededor de las TE

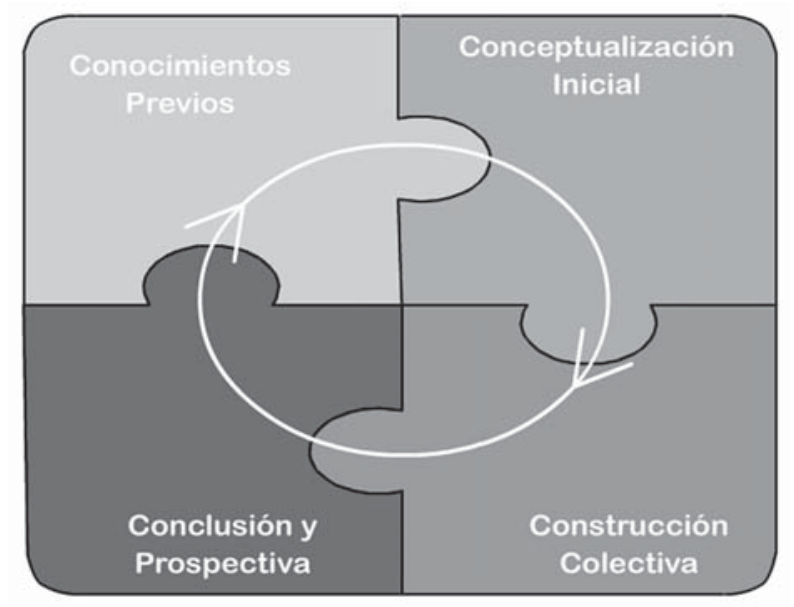

Luego de que todas las TE han sido abordadas dentro de la asignatura y los estudiantes han logrado generar de forma colectiva: un marco conceptual común sobre cada TE, sus reflexiones sobre las implicaciones de incorporar las Tecnologías Emergentes en la educación y de que han tomado una postura crítica y reflexiva sobre los aspectos a tener en cuenta a la hora de integrar TE a sus procesos educativos.

La asignatura propone un giro total en el ambiente de aprendizaje utilizado, pasa de ser un ambiente en donde se fomenta la reflexión y construcción colectiva a ser un ambiente en donde se favorecen los procesos de reflexión y metacognición en los estudiantes. Entendida esta última como el grado de conciencia que tenemos acerca de nuestras propias actividades mentales, es decir, de nuestro propio pensamiento y aprendizaje (ANTONIJEVICK; CHADWICK en VARGAS; ARBELÁEZ, 2002).

Para esto, se propone que los estudiantes trabajen de forma individual, identificando los conocimientos adquiridos, sus posturas y percepciones frente a las TE y que diseñen una estrategia didáctica que integre una o varias TE en sus contextos educativos. En él intervienen el docente como asesor académico, los estudiantes como protagonistas del proceso, a través de la reflexión y conceptualización y diseño de un ambiente de aprendizaje que integre una o varias TE. A continuación se presenta la estrategia utilizada. 


\section{Estrategia para la reflexión y metacognición de los estudiantes alrededor de las TE abordadas}

Tal y como se expuso al final del apartado anterior, una vez han sido abordadas todas las TE seleccionadas dentro del curso, en la segunda parte de la asignatura los estudiantes deben diseñar una estrategia didáctica que permita la integración de una de las TE en la educación básica, media o superior.

Para esto, se ha diseño una estrategia didáctica que tiene tres fases. La primera de ellas está encaminada a que los estudiantes identifiquen sus conocimientos a través de una actividad de interiorización, en la que a través de preguntas orientadoras los estudiantes realizan una autoevaluación de sus conocimientos alrededor de las temáticas abordadas, esto con el fin de identificar aquellas que por su nivel de conocimiento puedan ser utilizadas en la siguiente fase.

La segunda fase pretende que los estudiantes reflexionen sobre los diferentes aspectos que deben tener en cuenta a la hora de integrar una o varias TE dentro de un ambiente de aprendizaje mediado por TIC. Para tal fin, con las orientaciones del docente de la asignatura cada uno de los estudiantes realiza un mapa mental en donde, a partir de un proceso reflexivo, identifica un problema o necesidad educativa, luego, determina los principales aspectos de su contexto educativo, las diferentes concepciones pedagógicas en las que está soportado su ambiente de aprendizaje, las posibles estrategias didácticas que utilizará, los procesos que fomentará en sus estudiantes y, por último, reflexiona sobre los fines que tendrán las TIC y sobre cual o cuales de las TE abordadas pueden apoyar su ambiente de aprendizaje.

La última fase pretende que el estudiante, con la asesoría del docente de la asignatura, diseñe y describa de forma detallada un ambiente de aprendizaje que pueda ser integrado a su contexto educativo y que utilice de forma pedagógica una o varias TE.

\section{Resultados}

Luego de ofrecer la asignatura electiva en el programa de Maestría en Informática Educativa de la Universidad de La Sabana en el periodo académico 20102012, se obtuvieron los siguientes resultados:

Durante todo el semestre los estudiantes reconocieron, identificaron y reflexionaron sobre los diferentes aspectos que se deben tener en cuenta a la hora de integrar alguna de las siguientes Tecnologías Emergentes: Computación en la Nube, Inteligencia Colectiva, Mashups de Datos, Webs en Colaboración y Redes Sociales, en diferentes entornos educativos. 
Como resultado de este proceso, los estudiantes lograron desarrollar diferentes productos, tales como definiciones sobre la concepción de cada una de estas tecnologías desde el punto de vista educativo, posibles usos y aplicaciones académicas, ventajas y desventajas, así como determinar los principales aspectos que se deben tener en cuenta a la hora de diseñar estrategias o ambientes de aprendizaje que integren este tipo de tecnologías.

A continuación, se mostraran algunas de las definiciones propuestas y posibles usos reportados por los estudiantes:

a) Definición de Computación en la Nube desde el punto de vista académico por parte de los estudiantes:

Plataforma altamente escalable que elimina limitantes actuales a nivel de recursos de hardware y software, permitiendo el diseño de estrategias didácticas que utilizan recursos en internet y que contribuyen a los procesos de enseñanza y aprendizaje por medio de herramientas de fácil manejo; la nube potencia la igualdad de acceso a recursos, fomenta el trabajo colaborativo y estimula la innovación por medio de robustas aplicaciones web fáciles de utilizar.

b) Definición de Mashup de Datos desde el punto de vista académico, propuesta por un estudiante:

Entorno web personalizado, diseñado y utilizado por docentes y/o estudiantes, el cual reutiliza las características tecnológicas de otros entornos web preexistentes, cambiando sus objetivos de uso gracias la interoperabilidad que permiten los mismos, para la conformación de ambientes u objetos virtuales de aprendizaje de forma novedosa, flexible y sencilla.

c) Definición de Webs Colaborativas desde el punto de vista académico, propuesta por un estudiante:

[...] de esta manera las redes colaborativas mediadas por TIC son aquellos grupos de personas, en algunos casos de unos cuantos y en otras de muchos, que se reúnen a través de un tema en particular para conocerlo, interpretarlo y entenderlo y de esa manera trabajando en colaboración generar comunidad y conocimiento.

d) Definición de Inteligencia Colectiva desde el punto de vista académico, propuesta por un estudiante:

Es el trabajo y/o esfuerzo de un colectivo que beneficia al mismo a través de la consolidación de una idea, acción o producto, haciendo de este grupo especial y único. La IC es donde los individuos trabajan en pos del colectivo y no personal, teniendo un tema y propósito en común dándose hoy en un espacio que puede ser el ciberespacio actualmente apalancado en aplicaciones Web 2.0, que permiten relaciones variadas con jerarquías dinámicas, teniendo en estas la propia regulación de grupo.

e) Posibles usos de la Computación en la Nube en la educación: 
La computación en la nube permitirá un aprendizaje flexible en tiempo, modo y lugar para el estudiante, aprovechando mejor el tiempo y ahorrando los costos en la inversión educativa. Así mismo, facilitara el acceso a cualquier recurso sin necesidad de ser expertos en el uso de tecnologías. En el mismo sentido, las grandes prestaciones de almacenamiento y procesamiento serán escenarios ideales para realización de simuladores y utilización de sistemas expertos por parte de los docentes.

f) Posibles usos de los Mashup de Datos en la educación:

[...] los Mashups de Datos tienen diversas aplicaciones en la educación, pues, por sí dependen de la innovación del docente en su campo disciplinar y en actividades como presentación de contenidos y definición de secuencias y/o rutas de aprendizaje, entre otras. Permitiéndole a los docentes a través de esta innovación en la clase tener contenidos siempre actuales, interactivos y potenciadores de la participación del estudiante en las clases.

Me encontré este ejemplo de mashup que puede ser llevado a los procesos educativos: Histourist (http://www.histourist.com/) es un Mashup Semántico que ofrece una enciclopedia multimedia geolocalizada de lugares históricos. Los artículos se preparan mediante una combinación de editores y robots de software que explotan los recursos on-line en fuentes definidas como "confiables" (BBC, National Geographic, DBpedia, The History Channel, etc.) y en particular los servicios de la web semántica para enriquecer los artículos con videos, fotos, bibliografía, y clasificarlo en las taxonomías del servicio.

g) Posibles usos de las Webs Colaborativas en educación:

Web Colaborativa enriquecida con la escritura colaborativa (COBO; PARDO, 2007): Si bien como principal exponente encontraríamos en Wikipedia, es importante resaltar el hecho de cómo estefactor puede enriquecer los procesos de aprendizaje en los estudiantes, propiciandola elaboración y reelaboración de nuevos conceptos, dando cuenta del estado del arte de algo... fomentando la crítica, el debate, el auto-aprendizaje $[\ldots]$.

Aprovecharla inteligencia colectiva en la web colaborativa (COBO; PARDO, 2007; LEVY, 2007): Cambiar la actitud pasiva del estudiante a convertirloen un actor activo que pueda evidenciar tendencias, que amplié su visión delmundo más allá de los contenidos y dinámicas propuestas por el docente.

Webs colaborativas que propician la conformación de comunidades virtuales de aprendizaje (SALINAS, 2003), [...] bajo este esquema el concepto de web colaborativa toma otro estatus, donde docentes y estudiantes generan sentidos de pertenencia e identidad, propiciando así esa interacción social que se da enlos escenarios presenciales, permitiendo al estudiante asumir diversos roles y preocupaciones que superen los contextos y metas de la clase.

Ahora bien, a partir de las propuestas realizadas por los estudiantes y del proceso realizado en la asignatura, se proponen las siguientes definiciones desde el punto de vista académico para algunas de las tecnologías emergentes abordadas: 
Computación en la Nube: Tecnología escalable que brinda a docentes y estudiantes la posibilidad de acceder a recursos de almacenamiento, hardware y software, que facilitan el diseño e implementación de estrategias didácticas que propician el trabajo colaborativo, la construcción colectiva de conocimiento y la innovación.

Mashup de Datos: Tecnología que brinda a docentes y estudiantes la posibilidad de construir nuevo conocimiento a partir de la remezcla de recursos, información y conocimiento disponibles en internet. Así mismo, permite al docente el diseño de estrategias didácticas que favorezcan el desarrollo de competencias en manejo de información, el trabajo colaborativo y la construcción colectiva de conocimiento.

Web Colaborativa: Es una herramienta que brinda al docente la posibilidad de diseñar e implementar estrategias didácticas que favorezcan el intercambio de saberes entre los estudiantes, así como la construcción colectiva de conocimiento. Así mismo, apoyan el desarrollo de proyectos colaborativos, tales como la escritura colectiva de textos que contribuyen al desarrollo de competencias comunicativas en los estudiantes.

Inteligencia Colectiva: Mas que una herramienta o tecnología, es un concepto que invita al docente a diseñar estrategias didácticas que contribuyan al desarrollo de proyectos colaborativos que sean significativos para sus estudiantes, permitiéndoles así ser parte de un colectivo cuyas interacciones contribuyen al desarrollo de las competencias de sus integrantes y generan productos de conocimiento que van más allá de la suma de las capacidades individuales estos.

Asimismo, como producto del trabajo realizado por las parejas dentro del ambiente de aprendizaje, se diseñaron cinco estrategias de construcción colectiva de conocimiento, una por cada una de las TE abordadas. Dichas estrategias tenían un doble fin. El primero era permitir que los estudiantes sin compañía o seguimiento del docente lograran construir un Blog académico, producto del trabajo realizado durante las fases Construcción Colectivay Conclusión y Prospectiva.

Para elaborarlo, los estudiantes toman como materia prima el trabajo realizado por el New Media Consortium en el informe Horizon, sus concepciones previas, las indagaciones realizadas en internet y bases de datos especializados, así como las discusiones y conclusiones realizadas por el grupo. El resultado final era publicado por los líderes de cada temática, en el Blog del proyecto.

El segundo fin era que estas estrategias les sirvieran de ejemplo a sus compañeros, para apoyar su práctica docente. Las evidencias de todo el proceso de construcción colectiva de conocimiento de los estudiantes quedaron registradas en un foro, dispuesto para tal fin en cada uno de los módulos de la asignatura, dentro de la plataforma virtual que es utilizada por la Universidad para apoyar el trabajo independiente de los estudiantes denominada Virtualsabana. En las figuras 3 y 4 puede observarse algunos de los foros. 


\section{Figura 3}

Foro de construcción colectiva de conocimiento sobre Web Colaborativa

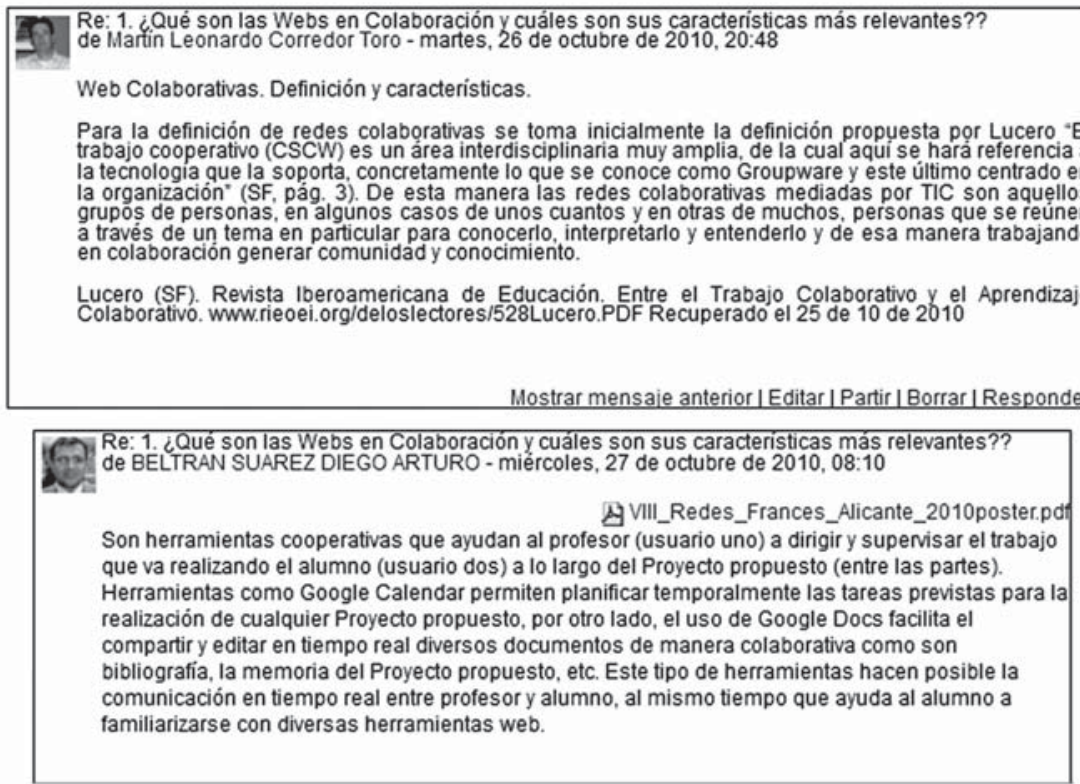

\section{Figura 4}

Foro de construcción de conocimiento sobre Computación en la Nube

Dándole forma a la nube de la educación superior

de ALEJANDRO EMILIO WLLS FONSECA-lunes, 13 de septiembre de 2010, 17:27

A Hignite_K_Katz_R_Yanosky_R_2010_Shaping_the_Higher_Education_Cloud_PUB9009.pdi Apreciados todos,

Este articulo es un muy buen resumen de los conceptos clave. Es producto de una conferencia que se llevó a cabo en febrero de este año. Es de notar la creación de un muy bonito término: cloudsourcing. Ahi les queda.

\section{Mostrar mensaje anterior /Editar /Partir /Borrar /Responder}

¿Y las desventajas?
de ALEJANDRO EMILIO WILLS FONSECA- lunes, 13 de septiembre de 2010, 17:07

Creo que no hemos tocado en detalle el tópico de las desventajas de la computación en la nube; para . el campo de la educación creo que serán las mismas que para los demás campos, con algunas particularidades. Les traje un articulo, bueno en realidad es un blog de Phil Morettini, Presidente de PJM Consulting, una firma especializada en tecnologia; es del 2009. y postula que todo el "bombo" en torno de la computación en la nube es un fenómeno ya visto. Evidentemente, la curva del bombo de Gartner es un modelo que nos muestra que toda tecnologia emergente sigue un camino parecido. Me interesa del articulo que ataca justamente lo que se nos presenta como mayor ventaja del modelo de negocio de cloud computing: la reducción de costos. Dice Morettini que si una mira la acumulación de costos a lo largo del tiempo, la reducción de transferencia al consumidor final no es tanta. Y se preocupa por los distribuidores del software, cuyos márgenes se van al piso, es decir, pone en tela de juicio la sostenibilidad del modelo. Estas inquietudes deben ser también parte de nuestra discusión. Mostrar mensaje anterior |Editar | Partir |Borrar |Responder

Re: Computacion en la Nube
de CACERES PEREZ GRACCE KAREN - martes, 14 de septiembre de 2010, $12: 49$

Computación en la nube - Reporte Horizont

Tomando algunas notas y reflexiones que arrojaron la construcción del Reporte Horizont, comparto algunos puntos tratados. 
Por otro lado, en la segunda parte de la asignatura los estudiantes debían diseñar un ambiente de aprendizaje que integrará una o varias de las TE abordadas en sus contextos educativos, los cuales van desde la educación básica, media, técnica y superior. Al final de la asignatura, se logró generar ocho ambientes de aprendizaje que integran una o varias Tecnologías Emergentes en diferentes contextos educativos.

\section{Conclusiones y prospectiva}

Luego de diseñar y orientar la asignatura tecnologías emergentes en educación en el segundo periodo de 2010, en la Maestría en Informática Educativa de la Universidad de La sabana, fue posible identificar que el ambiente de aprendizaje diseñado contribuyo al desarrollo de las competencias planteadas en la asignatura, gracias a que en éste tanto docentes como estudiantes asumieron con responsabilidad los roles planteados.

Particularmente, el papel que asumieron los líderes de cada temática, junto a las estrategias propuestas por cada uno de ellos resultaron fundamentales para el éxito del proceso, debido a que motivaron a sus compañeros a trabajar durante todo el semestre como un equipo interdisciplinario que buscaba comprender la naturaleza de las TE propuestas, sus usos y aplicaciones en contextos educativos, así como las ventajas y desventajas que podrían generar la incorporación de este tipo de tecnologías en sus contextos de práctica.

Asimismo, el rol que asumió el docente como orientador y facilitador de las dinámicas propuestas en el ambiente de aprendizaje, interviniendo solo para reencausar la discusión y la construcción de conocimiento por parte de los estudiantes, resulto ser muy importante, pues gracias a esto los estudiantes asumieron un papel protagónico durante el proceso.

Tal y como se puede evidenciar en las respuestas de los estudiantes cuando se les pregunto sobre el aporte realizado por el ambiente de aprendizaje

Estudiante 1: Por otra parte, la preparación y diseño de la estrategia estimula a los estudiantes a pensar en los usos educativos que pueden generarse de ella. Algo interesante es que al hacer este tipo de ejercicios el estudiante puede proyectar otro tipo de usos, hasta ahora no definidos o explorados antes.

[...] la metodología de la asignatura provoca cierto tipo de decisiones sobre la selección de lo que se desea indagar, es decir, son los estudiantes quienes escogen en que desean indagar con más profundidad.

Ahora bien, tal y como se indicó en la descripción del ambiente de aprendizaje en él participaron tres actores diferentes, el docente y los estudiantes como agentes dinamizadores del proceso y las TIC como agente pasivo del proceso, sin embargo, 
la función dada a las TIC dentro de este ambiente de aprendizaje como mediador de los procesos de interacción, comunicación, intercambio de saberes, conceptualización, y construcción colectiva de conocimiento por parte de los estudiantes, contribuyo a que los estudiantes lograran.

Asimismo, el profesor-autor de este trabajo confirmo que, en este tipo de ambientes, "los estudiantes son quienes trazan los límites del proyecto y que es función y deber del docente potenciar que estos sean alcanzados por sus estudiantes, para lograrlo, este no solo debe cambiar su rol en el aula, sino que debe permitir que sus estudiantes lo hagan" (BOUDE FIGUEREDO, 2011, p. 120).

Además, fue posible constatar que en este tipo de ambientes la función del docente, además de orientar las dinámicas que ocurren, es fomentar de forma constante la participación activa de los estudiantes, pues de esto depende en gran parte el éxito o fracaso de la misma.

Ahora bien, uno de los aspectos a mejorar en este ambiente de aprendizaje está relacionado con el rol que debe cumplir el docente durante la fase de construcción colectiva, pues los estudiantes tienen dificultades en coordinar el proceso de su participación, quedando en los foros las participaciones individuales de los estudiantes y no el resultado de un proceso de construcción colectiva.

Debido a la limitante de tiempo que tiene esta asignatura, no es posible que los estudiantes pongan en marcha el ambiente de aprendizaje diseñado. Lo cual puede generar una sensación de frustración en el estudiante y desmotivarlo en su proceso de diseño, ya que esta se ve más como un requiso que cumplir dentro de la asignatura y no como una ganancia para el contexto en el que se desenvuelve. Sin embargo, de los ocho ambientes de aprendizaje diseñados por los estudiantes, tres de ellos fueron puestos en práctica por parte de los estudiantes en su contexto educativo.

Los resultados obtenidos con esta experiencia sirvieron de base para que el profesor autor de este texto rediseñara el ambiente de aprendizaje y diseñará una propuesta de investigación formal sobre el mismo, que se está llevando a cabo en durante el 2011 y que se encuentra actualmente en la fase de análisis de los resultados.

\section{Referencias}

BOUDE FIGUEREDO, O. Proceso de construcción de un material educativo multimedia basado en casos. In: CONGRESO IBEROAMERICANO DE INFORMÁTICA EDUCATIVA, 9., Caracas, 2008. Actas... Caracas: Rede Iberoamericana de Informatica Educativa, 2008. 
BOUDE FIGUEREDO, O. Pediatic: desarrollo de competencias en TIC a través del aprendizaje por proyectos. Revista de Educación Médica Superior, La Habana, v. 25 n. 2, 2011.

CASTELLS, M. La era de la información: economía, sociedad y cultura. Madrid: Alianza, 1997. v. 1.

CENTRO DE TECNOLOGÍAS PARA LA ACADEMIA. Plan de Desarrollo 2011-2015; documento de trabajo. Bogotá: Universidad de La Sabana, 2010.

CHIAPPE, A; BOUDE FIGUEREDO, O. Proyecto de maestría; documento interno de trabajo de la Maestría en Informática Educativa. Bogotá: Universidad de La Sabana, 2010.

COBOROMANÍ, C.; PARDOKUKLINSKI, H. Planeta Web 2.0: inteligencia colectiva o medios fastfood. Barcelona: Uvic; México, DF: Flacso, 2007.

COLL, C. Aprender y enseñar con las TIC: expectativas, realidad y potencialidades. 2008. Disponível em: <http://escritoriodocentes.educ.ar/datos/recursos/articulos/aprender_ y_ensenar_con_tic.pdf $>$. Acesso em: nov. 2011.

COLOMBIA. Ministerio de Educación Nacional. Plan decenal de educación 2006-2016. Bogotá: MEN, 2006. Disponível em: <http://www.plandecenal.edu.co/html/1726/articles-166057_archivo_pdf.pdf >. Acesso em: 26 ago. 2011.

GONZÁLEZ TIRADO, R.; GONZÁLEZ MAURA, V. Diagnóstico de necesidades y estrategias de formación docente en las universidades. Revista Iberoamericana de Educación, Madrid, v. 43, n. 6, 2007.

HARGREAVES, A. Enseñar en la sociedad del conocimiento. Madrid: Octaedro, 2003.

HINOJO LUCENA, F.; FERNÁNDEZ MARTÍN, F.; ANZAR DÍAZ, I. Las actitudes de los docentes hacia la formación en tecnologías de la información y comunicación (TIC) aplicadas a la educación. Contextos Educativos: revista de educación, La Rioja, v. 5, p. 253-270, 2002.

JARAMILLO, P. et al. Informática, todo un reto. Ambientes de aprendizaje en el aula de informática: ¿Fomentan el manejo de la información? Bogotá: Uniandes, 2005.

JARAMILLO, P.; CHIAPPE, A.; SANCHEZ, J.O. Documento maestro de la Maestría en Informática Educativa; documento de trabajo. Bogotá: Universidad de La Sabana, 2007.

LEVY, P. Cibercultura: la cultura de la sociedad digital. Barcelona: Anthropos, 2007.

LLORENTE CEJUDO, M. Aspectos fundamentales de la formación del profesorado en TIC. Pixel-Bit: revista de medios y educación, Sevilla, n. 31, p. 121-130, 2008. 
MAESTRÍA EN INFORMÁTICA EDUCATIVA (Bogotá). Proyecto de Maestría. Bogotá: Universidad de La Sabana, 2010.

MARCELO, C. Aprender a enseñar para la Sociedad del Conocimiento. Revista Complutense de Educación, Madrid, v. 12, n. 2, p. 531-593, 2001.

NONAKA, I.; TAKEUCHI, H. La organización creadora de conocimiento: cómo las compañias japonesas crean la dinámica de la innovación. México, DF: Oxford University, 1999.

PROYECTO TUNING. Tuning Educational Structures in Europe: la contribución de las universidades al proceso de Bolonia. 2003. Disponível em: $<$ http://tuning.unideusto. org/tuningeu/images/stories/template/General_Brochure_Spanish_version.pdf $>$. Acesso em: oct. 2010.

SALINAS, J. Comunidades virtuales y aprendizaje digital. Edutec'03, artigo presentado no 6. Congreso Internacional de Tecnología Educativa y NNNT aplicadas a la educación: gestión de las TIC en los diferentes ámbitos educativos, realizado en la Universidad Central de Venezuela, 2003.

SALINAS, J. Innovación docente y uso de las TIC en la enseñanza universitaria. Revista de Universidad y Sociedad del Conocimiento, Barcelona, v. 1, n. 1, p. 1-16, 2004.

UNESCO. ICT Competency Standards for Teachers. 2008. Disponível em: <http://cst. unesco-ci.org/sites/projects/cst/default.aspx>. Acesso em: 6 oct. 2010.

VARGAS, E.; ARBELÁEZ, M. Consideraciones teóricas acerca de la metacognición. Revista Ciencias Humanas, Pereira, Colombia, n. 28, 2002. Disponível em: <http:// www.utp.edu.co/ chumanas/revistas/revistas/rev28/vargas.htm>. Acesso em: 15 feb. 2011.

Recebido em 9 de novembro de 2011.

Aprovado em 9 de janeiro de 2013. 